\title{
Osificación del Ligamento Estilohioideo en 3.028 Radiografías Panorámicas Digitales
}

\author{
Ossification of the Stylohyoid Ligament in 3028 Digital Panoramic Radiographs
}

\author{
Ivonne Garay* \& Sergio Olate**
}

GARAY, I. \& OLATE, S. Osificación del ligamento estilohioideo en 3.028 radiografías panorámicas digitales. Int. J. Morphol., 31(1):3137, 2013.

RESUMEN: La mayoría de los diagnósticos de procesos estiloides elongados y osificaciones de ligamentos estilohioideos corresponden a hallazgos en el análisis de radiografías panorámicas. En la presente investigación se evaluaron 3028 Radiografías Panorámicas digitales, tomadas entre Junio de 2009 y Junio de 2011. Se registraron las osificaciones de ligamento estilohiodeo de acuerdo a sexo, edad y bilateralidad. El análisis de datos se desarrolló con el Test estadistico Chi cuadrado, con un nivel de significancia estadística a $<0,05$. En las imágenes evaluadas se encontró una prevalencia de osificaciones de un 8,42\%, siendo más frecuentes en individuos de sexo femenino, osificaciones bilaterales y con patrón discontinuo. En cuanto a la edad se evaluó de acuerdo a grupos de edad menor o igual a 40 años y mayores de 40 años, encontrándose relación con la variable patrón de osificación. También se encontró relación entre las variables lateralidad y patrón de osificación. El presente estudio entrega información relevante para la odontología y la otorrinolaringología.

PALABRAS CLAVE: Radiografía panorámica; Síndrome de Eagle; Calcinosis.

\section{INTRODUCCIÓN}

El proceso estiloides, el ligamento estilohioideo y el asta menor del hueso hioides forman parte del aparato estilohiodeo, derivado del segundo arco branquial (cartílago de Reichert), el cual es conformado cuatro porciones: 1) Tímpanohial, se origina antes del nacimiento y se fusiona a la zona petrosa del hueso temporal para formar la porción timpánica del proceso estiloides y el osículo estapes. 2) Estilohial, se origina después del nacimiento y forma la porción media del proceso estiloides. 3) Ceratohial, genera el ligamento estilohioideo durante la etapa intrauterina, y 4) Hipohial, forma el cuerno menor y parte superior del cuerpo del hueso hioides (Tísner et al., 2003; RodríguezVázquez et al., 2006; Cantín et al., 2007).

El proceso estiloides es normalmente un hueso delgado, cilíndrico, ubicado inmediatamente en frente del foramen estilomastoideo y fusionado a la parte inferior del hueso temporal. Normalmente se ubica entre las arterias carótida externa e interna, medial a la arteria carótida externa y al músculo hiogloso presentando también tres músculos (estilofaríngeo, estilohioideo y estilogloso) y dos liga- mentos (estilohioideo y estilomandibular), insertados en él. La extremidad del proceso se continúa con el ligamento estilohioideo, que se extiende hasta el asta menor del hueso hioides (Monsour \& Young, 1986).

El tamaño promedio de este proceso estiloides es de $25 \mathrm{~mm}$ y cuando es mayor de $30 \mathrm{~mm}$ pasa a ser considerado elongado (Keur et al., 1986; Prabhu et al., 2007). El proceso estiloides elongado no da lugar a ningún tipo de dolor significativo, malestar o limitación del movimiento del cue1lo. A menudo permanece asintomático hasta que se descubre en las radiografías extraorales. El síndrome de Eagle implica dolor y malestar en la región cervicofacial que resultan específicamente de un proceso estiloides elongado (Mupparapu \& Robinson, 2005).

Se ha señalado que entre un $4 \%$ y $28 \%$ de la población posee procesos estiloides elongados, y de ellos, el $4 \%$ evidencia sintomatología (Fuentes et al., 2007; Radfar et al., 2008). En reportes previos también se observó que la elongación del proceso estiloides se presenta más en muje-

\footnotetext{
* Alumna del Programa de Magister en Odontológicas y Docente Unidad de Imagenología Oral y Maxilofacial, Universidad de La Frontera, Chile.

** Profesor Programa de Magister en Ciencias Odontológicas y Profesor Asistente Unidad de Cirugía Oral y Maxilofacial, Universidad de La Frontera, Chile.
} 
res, pero con mayor longitud en los hombres (Fuentes et al. 2007).

Se debe diagnosticar el síndrome de Eagle principalmente sobre la base de una evaluación clínica, incluyendo el historial médico del paciente y características del dolor, su duración y ubicación. La palpación digital del proceso estiloides en la fosa tonsilar reproduce y exacerba el dolor, y se indica alargamiento porque los procesos normales no son palpables. Estudios imagenológicos, tales como radiografías panorámicas, Tomografia Computarizada y la Tomografía Computarizada Cone Beam permiten determinar la longitud y la angulación del proceso estiloides así como la relación anatómica entre el proceso estiloides y el ligamento estilohioideo (Pagani et al., 2010; Rubio et al., 2010).

El objetivo de esta investigación fue identificar la prevalencia de osificación de ligamento estilohiodeo y proceso estiloides elongado mediante el análisis de radiografías panorámicas digitales.

\section{MATERIAL Y MÉTODO}

Selección de pacientes. Se diseñó un estudio descriptivo poblacional para evaluar 3028 radiografías panorámicas digitales de pacientes que asistieron a un centro radiológico privado de la ciudad de Temuco, Chile, para análisis y diag- nóstico de patologías vinculadas a implantología, ortodoncia, cirugía oral o diagnóstico general entre junio de 2009 y junio de 2011. Fueron incluidas todas aquellas radiografías panorámicas de sujetos sin discriminacion de edad o sexo.

De las 3028 radiografías panorámicas analizadas, 1838 correspondían al sexo femenino y 1190 al sexo masculino. Las edades de los pacientes en el momento de la toma de las radiografías panorámicas estaban en el rango de 3 a 90 años, con una media de 28 años (DE: 18.5 años) (Fig. $3)$.

Obtención de imágenes. Las radiografías panorámicas fueron obtenidas del equipo ortopantomógrafo Instrumentarium 0P200 D, Finlandia, 2008, a través del sowtware Cliniview XV en monitor LG Flatron de 19 pulgadas.

Las tomas radiográficas fueron realizadas por un técnico especialmente entrenado, con $\mathrm{kV}$ 66, $\mathrm{mA}$ 5-10 y un tiempo de exposición de 17,6 segundos. Las imágenes fueron evaluadas y aprobadas por un radiólogo con 5 años de experiencia; posteriormente el mismo radiólogo realizó las evaluaciones para determinar las variables del estudio.

Análisis de Imágenes. Se estudió la presencia de osificación de ligamento estilohioideo, así como sus características uni o bilaterales y patrón de osificación continuo o discontinuo. Se incluyeron aquellas imágenes donde se observaron procesos estiloides mayores a $30 \mathrm{~mm}$ (Fig. 1) y aquellas donde se observó osificación en distintas porciones del

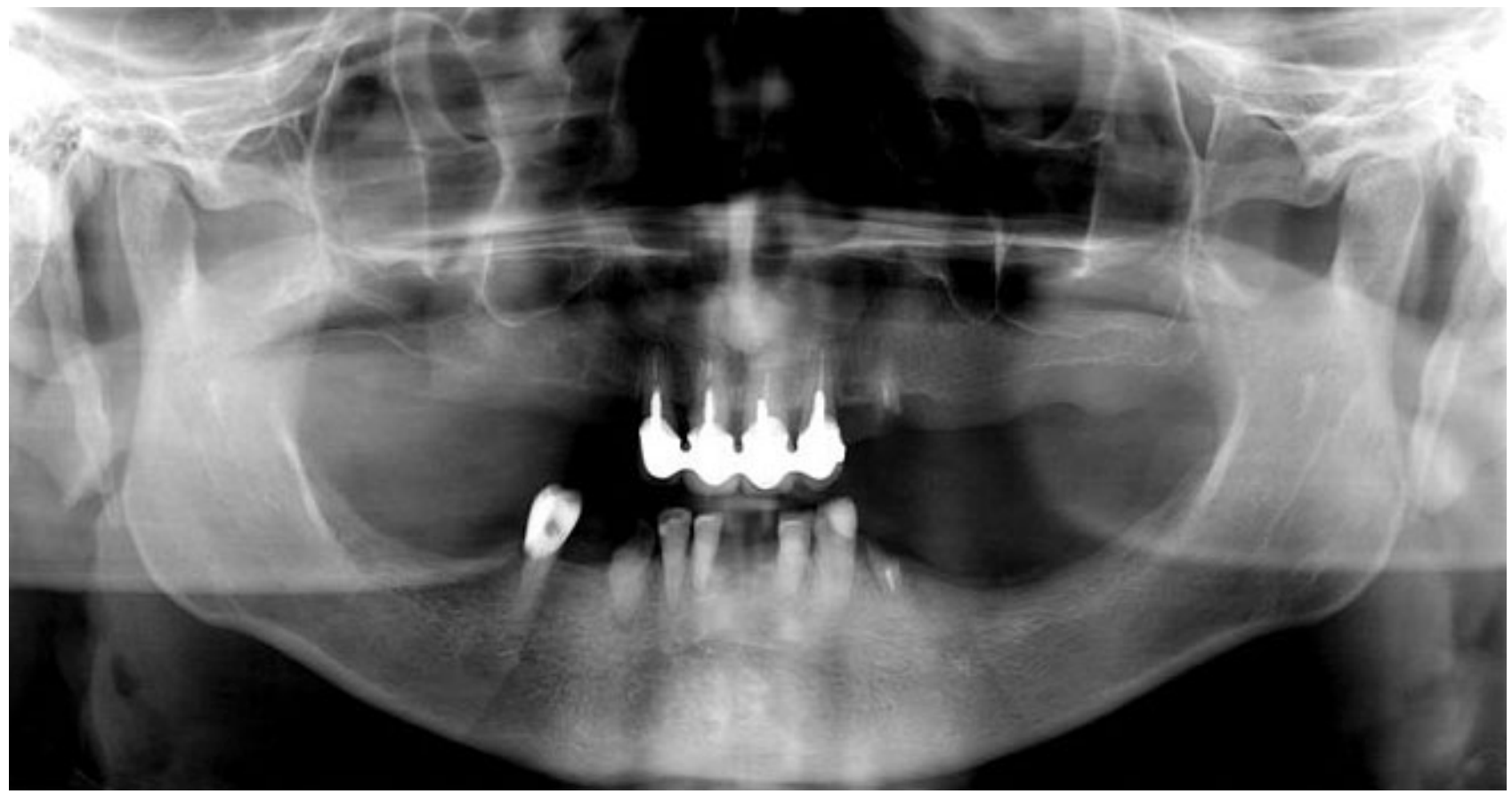

Fig. 1. Proceso estiloides elongado u osificación continua de ligamento estilohiodeo, bilateral. 


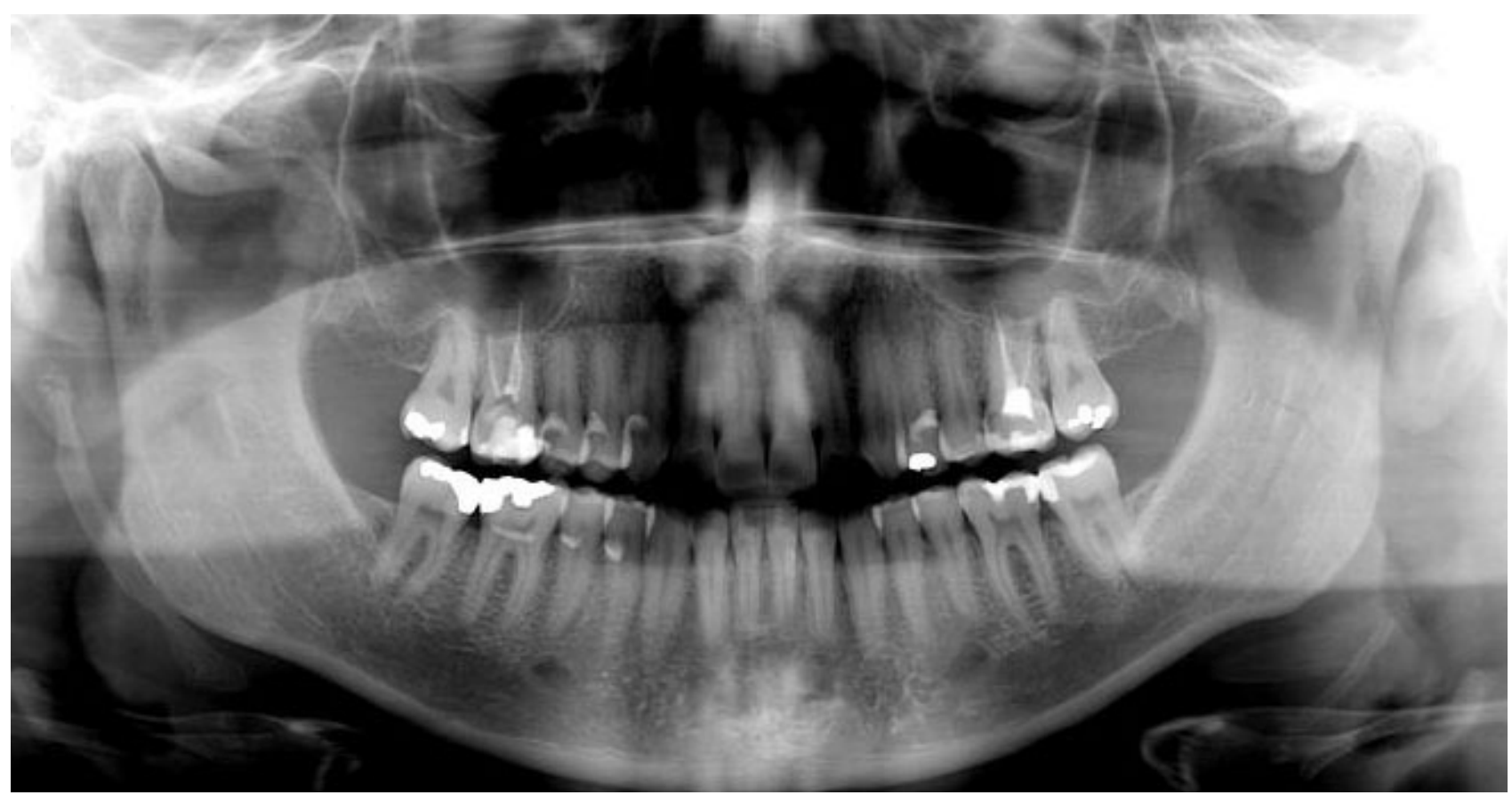

Fig. 2. Osificación unilateral derecha de ligamento estilohioideo, con patrón discontinuo. Nótese proceso estiloides de longitud normal.

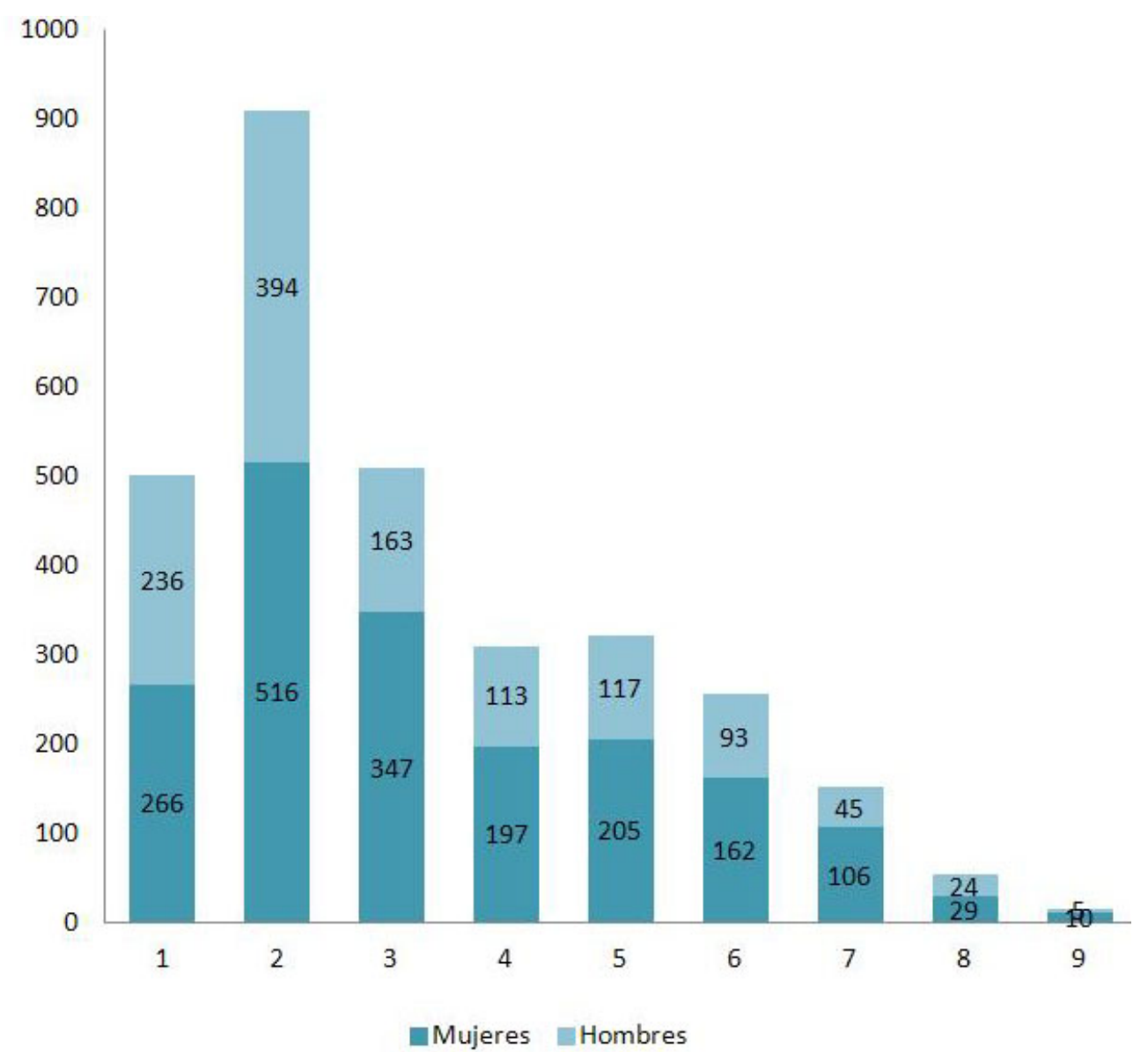

ligamento estilohioideo (Fig. 2). Se descartaron aquellas imágenes con diagnóstico dudoso por sobreproyección de estructuras o falta de nitidez.

Las radiografías panorámicas fueron observadas en pantalla completa, las áreas de baja radiopacidad compatibles con osificación de ligamento estilohioideo fueron evaluadas mediante una herramienta del software con magnificación $\mathrm{x} 2$.

Análisis estadístico. Se utilizó el software estadístico Stata 9.0/SE, aplicándose la prueba Chi cuadrado considerando un nivel de significancia estadística de $\mathrm{a}=0,05$.

Fig. 3. Distribución de edad y proporción de hombres y mujeres. 


\section{RESULTADOS}

Se encontró osificación de ligamento estilohiodeo en 255 individuos $(8,42 \%)$, de los cuales, $156(61,18 \%)$ correspondían a individuos de sexo femenino y $99(38,82 \%)$ a individuos de sexo masculino. La proporción de calcificaciones de acuerdo a grupos etarios se muestra en la Figura 4. De los individuos en que se encontraron calcificaciones, 164 individuos (64,31\%) eran de edad menor o igual a 40 años y $91(35,69 \%)$ eran individuos con edad mayor a 40 años.

La evaluación de osificación de ligamento estilohioideo en cuanto a sexo y grupo de edad no mostró significación estadística (Tabla I). Tampoco se observó relación entre las variables sexo y lateralidad (Tabla II); las otras variables estudiadas como el sexo, patrón de osificación y lateralidad no presentaron significancia estadística. Se encontraron diferencias estadísticamente significativas en cuanto a grupo etario y patrón morfológico $(p<0,001)$ así como también entre lateralidad y patrón morfológico $(\mathrm{p}=0,048)$ (Tabla III).

De las imágenes encontradas, no todas presentaban procesos estiloides con medidas mayores a $30 \mathrm{~mm}$, en muchos individuos se observaron distintos focos de osificación a lo largo del ligamento estilohioideo. En algunos casos de pacientes jóvenes se observó menor grado de radiopacidad, e incluso sobreproyección a nivel de ángulo mandibular.

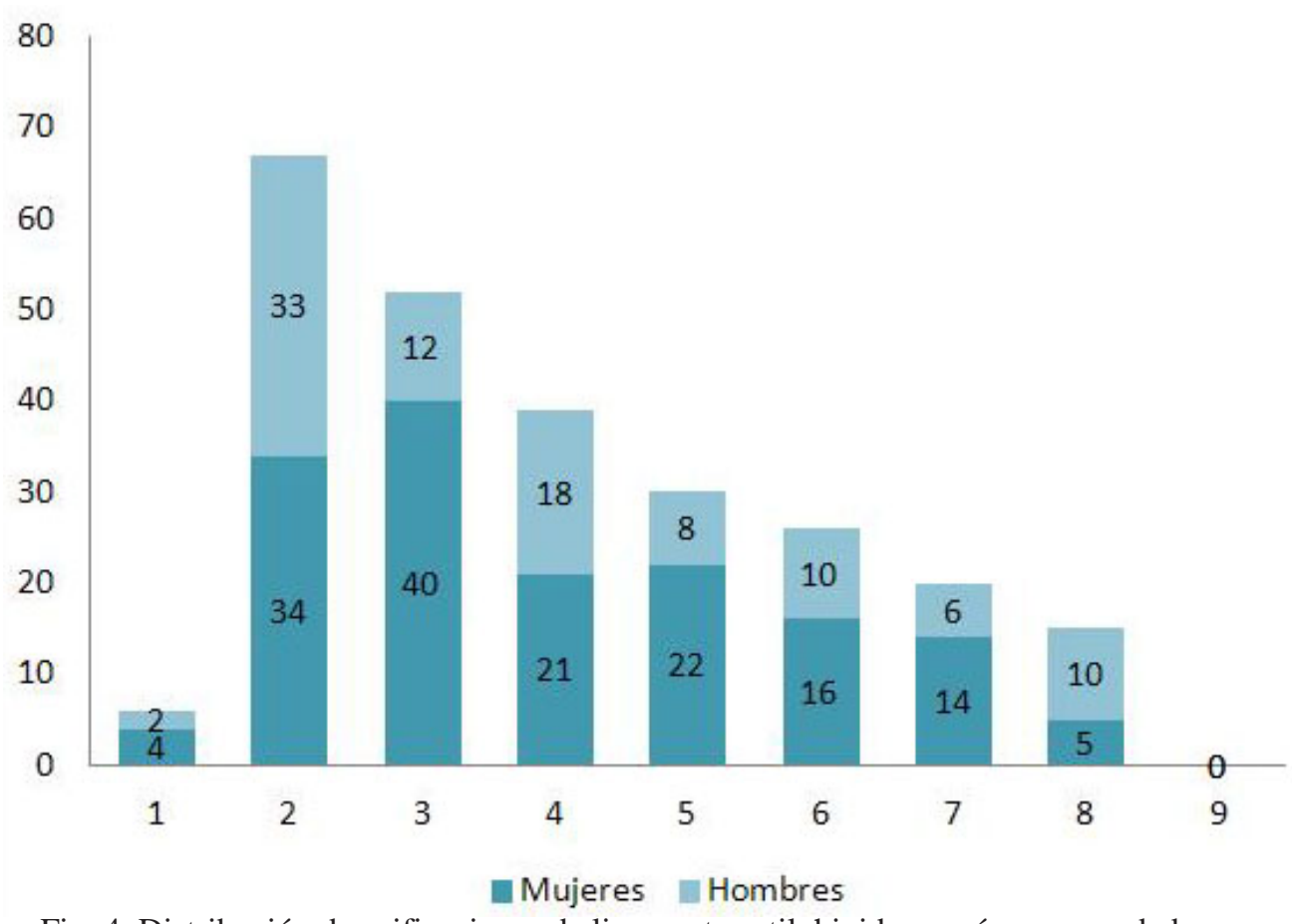

Fig. 4. Distribución de osificaciones de ligamento estilohioideo según sexo y edad.

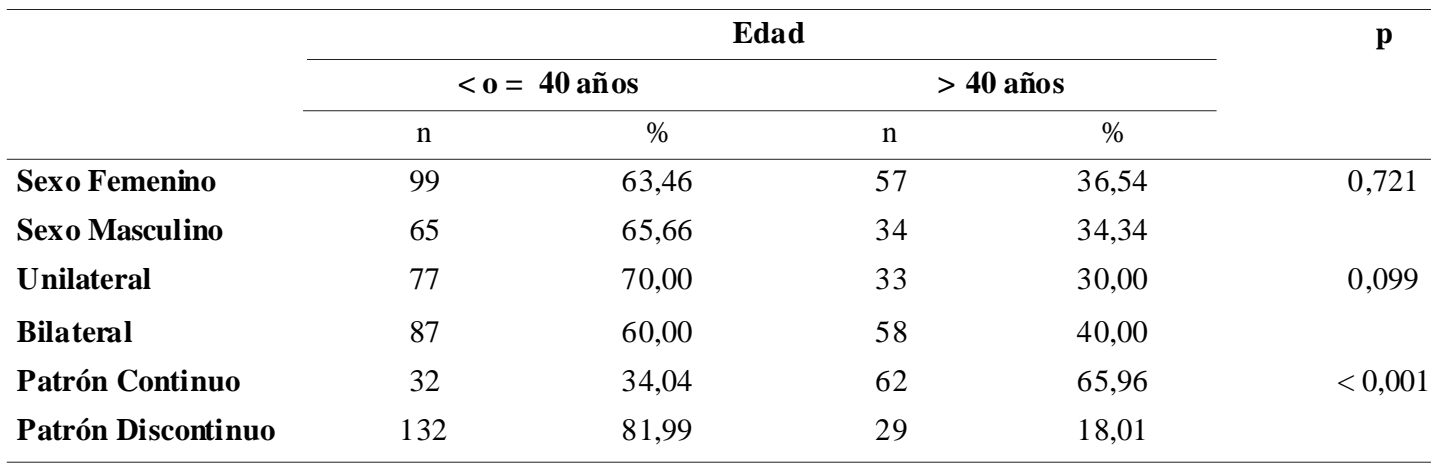




\begin{tabular}{|c|c|c|c|c|c|}
\hline \multirow{3}{*}{ Lateralidad } & \multicolumn{4}{|c|}{ Sexo } & \multirow[t]{3}{*}{$\mathbf{p}$} \\
\hline & \multicolumn{2}{|c|}{ Femenino } & \multicolumn{2}{|c|}{ Masculino } & \\
\hline & $\mathrm{n}$ & $\%$ & $\mathrm{n}$ & $\%$ & \\
\hline Unilateral & 61 & 55,45 & 49 & 44,55 & 0,102 \\
\hline Bilateral & 95 & 65,52 & 50 & 34,48 & \\
\hline Continuo & 63 & 67,02 & 31 & 32,98 & 0,143 \\
\hline Discontinuo & 93 & 57,76 & 68 & 42,24 & \\
\hline
\end{tabular}

\begin{tabular}{lcccccc}
\hline & \multicolumn{5}{c}{ Lateralidad } & \multirow{2}{*}{ p } \\
\cline { 2 - 5 } Patrón de & \multicolumn{3}{c}{ Unilateral } & \multicolumn{2}{c}{ Bilateral } & \\
\cline { 2 - 5 } osificación & $\mathrm{n}$ & $\%$ & $\mathrm{n}$ & $\%$ & \\
\hline Continuo & 33 & 35,11 & 61 & 64,89 & 0,048 \\
Discontinuo & 77 & 47,83 & 84 & 52,17 & \\
\hline
\end{tabular}

\section{DISCUSIÓN}

Actualmente no existe claridad en cuanto a las causas que originan osificación de ligamento estilohioideo. En la mayoría de los animales vertebrados el aparato hioideo se encuentra osificado siendo formando por un distinto número de huesos. En el hombre solamente son identificables los extremos de dicha cadena, es decir, el proceso estiloides y el asta menor del hioides. Probablemente, debido a esto existe la posibilidad de encontrar aparatos hioideos con un mayor grado de osificación (Tísner et al.).

Steinmann propuso tres teorías para justificar el proceso de osificación: 1) Teoría de la hiperplasia reactiva: los traumatismos debidos al sistema carotídeo en contacto con el ligamento estilohioideo, sobre todo en las rotaciones de la cabeza, hacen que restos de tejido conectivo se transformen en fibrocartílago precursor de osificación posterior, 2) Teoría de la metaplasia reactiva: como resultado de una actuación postraumática aberrante y 3) Teoría de la variación anatómica, sin evidencia de traumatismos sobre el ligamento estilohioideo.

En nuestro estudio fueron considerados tanto los procesos estiloides elongados como las osificaciones de ligamento estilohoideo en distintos puntos de su trayecto hasta el hueso hioides. Coincidimos con lo reportado por Tísner et al. quienes reportaron la presencia de aparatos con osificación parcial o incompleta que se corresponden con un proceso estiloides elongado y que es el hallazgo más frecuente.
Aparatos totalmente osificados son menos frecuentes, donde la variedad más encontrada lo formarían 3 huesos distintos: el hueso estilo-hial ocupa el lugar del proceso estiloides; el ceratohial el lugar del ligamento estilohioideo, y el hueso hipo-hial ocupa el lugar del asta menor del hioides. Esto concordaría con el aspecto seudoarticulado que tienen algunas de las imágenes encontradas, así como también, aquellas osificaciones parciales de la porción media del ligamento donde no se observó presencia de proceso estiloides elongado.

La prevalencia de elongación del proceso estiloides se encuentra entre el $4 \%$ y $28 \%$ de la población general (Radfar et al.; Fuentes et al.; Monsour et al.). Los resultados de la presente investigación difieren a lo reportado por Fuentes et al. quienes encontraron una prevalencia de 3,6\% en radiografías panorámicas convencionales, en este estudio realizado con radiografías panorámicas digitales se encontró una prevalencia de 8,42\%. Estas diferencias pueden deberse a un mayor tamaño de la muestra, la posibilidad de observar radiografías amplificadas en un monitor, modificar las características de la imagen digital que permitan identificar bajos grados de osificación de ligamento y el uso de herramientas de medición más precisas del software utilizado.

El porcentaje de osificación de ligamento estilohioideo y proceso estiloides elongado encontrado en nuestro estudio, es inferior a lo reportado por Radfar et al., 
quienes encontraron una prevalencia de $22 \%$ en un total de 1000 radiografías panorámicas. Esta diferencia de porcentajes se incrementa al comparar nuestros resultados con en el estudio de Rubio et al., quienes observaron una prevalencia de un $61 \%$ en una muestra de 80 individuos, analizados mediante Cone Beam CT. Sin embargo, en el estudio mencionado no se reporta la relación estadística de los resultados.

Nuestros resultados coinciden con los estudios de Correl et al. y Fuentes et al., quienes señalaron que esta alteración se presenta con mayor frecuencia en el sexo femenino; en el presente estudio se encontró que un 61,18\% de las osificaciones correspondían a mujeres, pese a esto, no se encontró significación estadística entre la presencia de osificación con la variable sexo. Diferencias fisiológicas entre hombre y mujer podrian sugerir alguna explicación a estas diferencias.

Castillo et al., clasifica a estas alteraciones como elongadas (al evidenciar una imagen del proceso calcificado de forma continua) y fragmentadas, con dos variantes de morfología (segmentada y pseudoarticulada) y concluyen que este patrón fragmentado es el más frecuente. Nuestros resultados coinciden con estos autores en cuanto a que el patrón segmentado o discontinuo sería más frecuente, puesto que de las osificaciones encontradas, 161 correspondían a esta forma $(63,14 \%)$. Se encontró además diferencias estadísticamente significativas entre esta variable con grupo etario y lateralidad. Si bien es cierto, en nuestra investigación se encontraron osificaciones en todos los grupos etarios, se encontró un mayor número de osificaciones discontinuas en individuos con edades menores o igual a 40 años, a diferencia de los individuos mayores de 40 años donde se observó osificaciones continuas. Esto último coincidiría con lo reportado por Rubio et al., y Fuentes et al., en cuanto a que el grado de osificación aumentaría en los grupos de mayor edad. Sin embargo, nuestro estudio difiere a lo encontrado por Fuentes et al., quienes observaron que el patrón continuo fue más frecuente. En relación a la lateralidad, observamos una mayor frecuencia de osificaciones bilaterales, esto también difiere con lo reportado por Fuentes et al., y coincide con lo reportado por Tísner et al.

En la presente investigación no se realizaron mediciones de longitud de osificaciones que permitieran realizar comparaciones en cuanto a género, aunque Fuentes et al. concluyen que se observaría una mayor longitud de las osificaciones en individuos de sexo masculino. Por otro lado, en nuestro estudio tampoco fueron considerados los aspectos clínicos que pudieran asociar la presencia de osificaciones de ligamento estilohioideo con Síndrome de Eagle.
Cabe destacar que dada la variabilidad de los estudios en lo que se refiere a la frecuencia de estas osificaciones, las asociaciones encontradas y el origen de causas no precisadas, se hace necesario realizar nuevas investigaciones, en las que se puedan utilizar otros estudios de imagen de mayor precisión como la tomografia computarizada cone beam. Por otro lado, creemos que estudios que permitan evaluar cambios de la osificación en el tiempo, como por ejemplo estudios de cohorte, contribuirían enormemente, tanto a odontólogos como a otorrinolaringólogos, a establecer los factores que influyen en la osificación de ligamento estilohioideo y elongación de proceso estiloides, que por cierto, consideramos sólo un hallazgo radiográfico o una variante anatómica y no patológica a menos que esté asociada a sintomatología dolorosa o indicativa de Síndrome de Eagle.

GARAY, I. \& OLATE, S. Ossification of the stylohyoid ligament in 3028 digital panoramic radiographs. Int. J. Morphol., 31(1):3137, 2013.

SUMMARY: Most diagnoses of elongated styloid processes and ossification of stylohyoideus ligaments correspond to findings in the analysis of panoramic radiographs. In the present study we evaluated 3028 digital panoramic radiographs, taken between June 2009 and June 2011. Stylohyoideus ligament ossifications were recorded according to sex, age and bilaterality. Data analysis was carried out with the chi square test, with a statistical significance level a $<0.05$. In the images assessed we found a prevalence of $8.42 \%$ of ossifications, which was more frequent in female subjects, and bilateral ossification discontinuous pattern. In terms of age we evaluated according to age group less than or equal to 40 and older than 40 years, as related to the variable pattern of ossification. We also found relation between laterality variables and patterns of ossification. This study provides information relevant to dentistry and otolaryngology.

KEY WORDS: Panoramic radiographies; Eagle síndrome; Calcinosis.

\section{REFERENCIAS BIBLIOGRÁFICAS}

Cantín, M.; Suazo, I.; Venegas, G. I.; Zavando, M. D. \& Muñoz, C. R. Síndrome de Eagle bilateral, reporte de un caso. Int. J. Odontostomat., 1(2):141-5, 2007.

Castillo, C. E.; Mantilla, J. C.; Sandoval, G. P. \& Ramírez, L. M. "Síndrome de Eagle: Tomografía del proceso estiloídeo elongado". Acta De Otorrinolaringología y Cirugía de Cabeza y Cuello., 31(3), 2003.

Correl, R.; Jensen, J.; Taylor, J. \& Rhyme, R: Mineralization of the 
stylohyoid - stylomandibular ligament complex: A radiographic incidence study. Oral Surg., 48:286, 1979.

Fuentes, R.; Oporto, G.; Garay, I.; Bustos, L.; Silva, H. \& Flores, $\mathrm{H}$. Proceso estiloides en una muestra de radiografías panorámicas de la ciudad de Temuco-Chile. Int. J. Morphol., 25(4):729-33, 2007.

Keur, J. J.; Campbell, J. P.; McCarthy, J. F. \& Ralph, W. J. The clinical significance of the elongated styloid process. Oral Surg. Oral Med. Oral Pathol., 61(4):399-404, 1986.

Monsour PA, Young WG. Variability of the styloid process and stylohyoid ligament in panoramic radiographs. Oral Surg. Oral Med. Oral Pathol., 61(5):522-6, 1986.

Mupparapu, M. \& Robinson, M. D. The mineralized and elongated styloid process: a review of current diagnostic criteria and evaluation strategies. Gen. Dent., 53(1):54-9, 2005.

Pagani, D.; Capaccio, P.; Balzani, A. \& Pignataro, L. Dysphagia and Submandibular Swelling. J. Am. Dent. Assoc., 141:108993, 2010

Prabhu, L. V.; Kumar, A.; Nayak, S. R.; Pai, M. M; Vadgaonkar, R.; Krishnamurthy, A. \& Madhan Kumar, S. J. An unusually lengthy styloid process. Singapore Med. J., 48(2):34-6, 2007.

Radfar, L.; Amjadi, N.; Aslani, N. \& Suresh, L. Prevalence and clinical significance of elongated calcified styloid processes in panoramic radiographs. Gen. Dent., 56(6):e29-32, 2008.

Rodríguez-Vázquez , J. F.; Merida-Velasco, J. R.; Verdugo-Lopez, S.; Sanchez-Montesinos, I. \& Merida-Velasco, J. A. Morphogenesis of the second pharyngeal arch cartilage (Reichert's cartilage) in human embryos. J. Anat., 208(2):17989, 2006.

Rubio, C.; Ganga, H. \& Guzmán, C. L. Analysis of the mediolateral insertion axis of the stylohyoid ligament regarding the sagittal plane of the skull in individuals of both sexes through the Cone Beam System: a retrospective study. Rev. Fac. Odontol. Univ. Antioquia, 22(1):33-9, 2010.

Rubio, C.; Ganga, H. \& Guzmán, C. L. Análisis de la longitud del ligamento estilohiodeo mediante sistema cone beam. Rev. CES Odont., 23(1):23-7, 2010.

Steinmann, E. P. A new light on the pathogenesis of the styloid syndrome. Arch. Otolaryngol., 91:171-4, 1970.

Tísner, J. V.; Tísner, G.; Abad, J. M. \& Abad, M. Stylohyoid process ossification: stylalgie of Aubin and Eagle's syndrome. Report of five cases. Rev. S. A. O.R.L., 6 (2): 5-12, 2003.

\author{
Correspondencia: \\ Prof. Dr. Sergio Olate \\ Unidad de Cirugía Oral y Maxilofacial \\ Universidad de La Frontera \\ Temuco - Chile.
}

Email: sergio.olate@ufrontera.cl

Recibido : 04-09-2012

Aceptado: 15-11-2012 